\title{
A test of Vitz and Todd's model of the judged complexity of straight-line figures
}

\author{
MICHAEL KUBOVY \\ Yale University, New Haven, Connecticut 06520 \\ and \\ JOSEPH TZELGOV \\ Ben Gurion University, Beer-Sheva, Israel
}

\begin{abstract}
Two sets of stimuli, each consisting of nine geometric patterns, were computer-generated: in one set, the patterns were squares which were partitioned randomly into rectangles; in the other, the patterns were squares which were partitioned randomly into polygons. Ten subjects gave magnitude estimates of the complexity of the stimuli. The Vitz and Todd (1971) index of complexity was not a consistently better predictor of estimated complexity than the ratio of the number of lines in each pattern to an index of symmetry. Moreover, the power function relating the complexity estimates of the rectangular partitions to the Vitz and Todd index was significantly different from the power function relating the complexity of the polygonal partitions to the Vitz and Todd index. It is concluded that the Vitz and Todd model requires major additional assumptions in order to be a candidate for a process model of pattern perception.
\end{abstract}

Vitz and Todd (1971) proposed a stochastic sampling model for the perception of simple geometric figures. According to this model, it is assumed that the visual system samples figural elements with replacement until all elements have been observed. Furthermore, it is assumed that plane figures have three classes of elements: line segments, angles, and areas. Angles and areas are self-explanatory here, but the notion of line segment must be explicated. A line segment can be bounded by two kinds of points, end points and intersection points. A line ending at another line does not, however, create an intersection point on the other line. Thus a + contains four segments, and a T only two. Each element has a measure. For instance, a given line segment has a length $\lambda_{\mathbf{i}}$, a given angle has an angular measure $\omega_{j}$, and a given area has a surface $\alpha_{\mathbf{k}}$. These measures are normalized within each class of element. Thus, segment $i$ is assigned a normalized measure

$$
\lambda_{\mathbf{i}} / \sum_{\mathbf{i}} \lambda_{\mathbf{i}}
$$

which is the probability of sampling segment $i$ on a given occasion. By analogy, the probabilities of

Requests for reprints should be sent to Michael Kubovy, Department of Psychology, Yale University, New Haven, Connecticut 06520. Portions of this paper are based on a thesis written by the second author under supervision of the first, and submitted in partial fultillment of the requirements for an M.A. degree at the Hebrew University of Jerusalem, Israel. We wish to thank A. Koriat for his advice, D. Rosenberg for writing the program which drew the patterns, M. Shamir for preparing the stimuli. sampling angle $\mathrm{j}$ or area $\mathrm{k}$ on a given occasion are

$$
\omega_{\mathrm{j}} / \sum_{\mathrm{j}} \omega_{\mathrm{j}} \text { and } \alpha_{\mathrm{k}} / \sum_{\mathrm{k}} \alpha_{\mathrm{k}}
$$

respectively. Finally, let $n_{\lambda}$ be the number of samples required to exhaust all the line segments of a figure during a particular sampling run; let $n_{\omega}$ and $n_{\alpha}$ be the number of glances required to exhaust all the angles and areas, respectively.

The perceived complexity of the figure is assumed to be a function of a linear combination of $n_{\lambda}, n_{\omega}$, and $n_{\alpha}$, where the number of samples needed to exhaust each class of figural elements is weighted by a degree of partial symmetry $\left(S_{\lambda}, S_{\omega}\right.$, and $\left.S_{\alpha}\right)$. We refer the reader to Vitz and Todd's (1971) paper for further details on the computation of partial symmetry. Since $\mathrm{n}_{\lambda}, \mathrm{n}_{\omega}$, and $\mathrm{n}_{\alpha}$ are random variables, Vitz and Todd developed a Monte Carlo method to obtain their expected values $\mathrm{E}\left(\mathrm{n}_{\lambda}\right), \mathrm{E}\left(\mathrm{n}_{\omega}\right)$, and $\mathrm{E}\left(\mathrm{n}_{\alpha}\right)$. Thus, the predicted complexity of a figure, according to Vitz and Todd's model, is $\mathrm{C}=\mathrm{E}\left(\mathrm{n}_{\lambda}\right) / \mathrm{S}_{\lambda}+\mathrm{E}\left(\mathrm{n}_{\omega}\right) / \mathrm{S}_{\omega}+$ $\mathrm{E}\left(\mathrm{n}_{\alpha}\right) / \mathrm{S}_{\alpha}$.

If some probabilities are very small (because of the large number of elements or because of the small measure of some of the elements), then the number of samples may become inordinately large. To avoid such spuriously large numbers, the Monte Carlo method allows for a stop rule criterion: the sampling process ceases after a certain number of attempts have failed to sample a new figural element.

Vitz and Todd have presented empirical evidence in favor of their model, finding high product-moment 
correlation coefficients (of the order of .96) between the computed values of $\mathrm{C}$ and the mean rank of judged complexity over a wide variety of closed polygonal forms (range: 3 to 23 sides).

The purpose of the present paper is to report a more general test of the model. Two sets of stimuli, different from the forms studied by Vitz and Todd, were generated by computer, using different algorithms so that the stimuli in different sets differed perceptually but were of the same order of magnitude of complexity in terms of $\mathrm{C}$. Two questions were asked: How well would $C$ predict the judged complexity of the forms? Would there be a systematic difference between the judged complexity of figures belonging to the two sets?

\section{METHOD}

\section{Stimuli}

Figure 1 shows the nine stimuli from each class used in the experiment. All the stimuli are partitions of a $100-\mathrm{mm}$ square. The sets differ in that Set $\mathrm{R}$ contains only rectangular partitions of the square, resembling the figures employed by Terwilliger (1963), whereas Set $\mathbf{P}$ contains only nonrectangular polygonal partitions of the square, resembling the figures employed by Vitz (1966). The stimuli were generated and plotted by computer, and presented to the subjects as a deck of 18 cards, $120 \mathrm{~mm}$ square. In the lower right-hand corner, a random permutation of the letters $w, x, y, z$ identified each card.

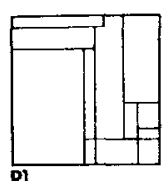

RI
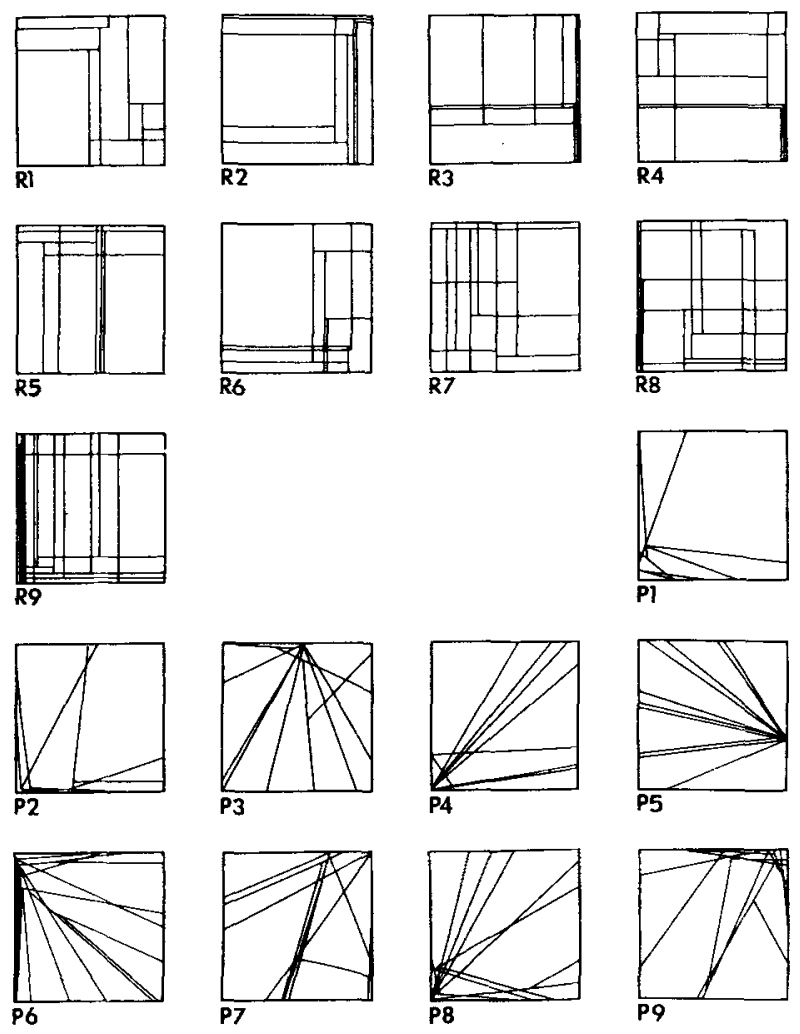

R4
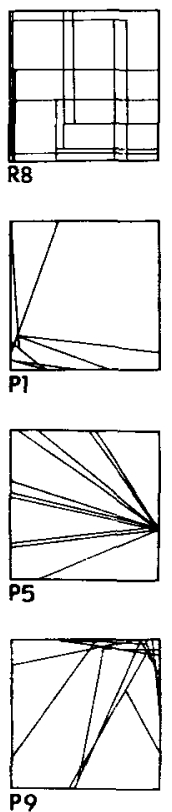

Figure 1. The 18 stimuli used in the experiment $(R$, rectangular set; $\mathbf{P}$, polygonal set).
Subjects

Ten volunteers (five of each sex) participated. All were undergraduates at the Hebrew University of Jerusalem.

\section{Procedure}

The subjects were run individually in sessions lasting about $25 \mathrm{~min}$. They were first asked to rank-order the stimuli by complexity. Then they were requested to give magnitude estimates of the complexity of the figures, assuming that the least complex figure's complexity was 10 . Subjects were encouraged to revise their ranking and magnitude estimates at any stage of the procedure, and to stop only after they were satisfied that the estimates reflected their judgments.

\section{RESULTS}

The general results are summarized in Table 1 and in Figure 2. Table 1 shows $C$ as calculated by Vitz and Todd's Monte-Carlo program ${ }^{1}$ (based on 1,000 sampling runs and a stop rule criterion of 75 , as recommended by Vitz and Todd) and the geometric mean of the complexity magnitude estimates. Figure 2 graphs on a $\log -\log$ plot the geometric mean of the magnitude estimates of complexity as a function of $\mathrm{C}$.

The following statements apply to a logarithmic transformation of the dependent and independent variables, since power functions are more likely to hold for magnitude estimates. Table 2 summarizes the results in greater detail. A simple linear regression equation accounts for an estimated $\mathrm{R}_{\mathrm{C}}^{2}=.7493$ of the variance in the geometric mean of the magnitude estimates. If, however, we compute a multiple regression equation with complexity as the first variable (continuous) and type of partition (rectangular vs. polygonal) as the second variable (categorical), we can account for $\mathrm{R}_{\mathrm{Cp}}^{2}=.8620$ of the variance. Thus we can increase the proportion of variance accounted for by .1127 by adding the type-of-partition variable. The effect of type of partition was found to be significant by an analysis of covariance after adjustment for complexity $[\mathrm{F}(1,15)$ $=12.25 ; \mathrm{p} \cong .0032]$. No significant difference was found between the within-set slopes $[\mathrm{F}(1,14)=2.13$; $\mathrm{p}=.16]$. Thus the data are best described by the two parallel regression lines shown in Figure 2, the intercept of the regression for Set $\mathrm{R}$ being lower than that for Set P.

The pattern of the group average is fairly representative of the individual subjects (see Table 2): for 6 of the 10 subjects, the intercept of the regression for Set $R$ was lower than that for Set $P$; for three (Subjects 2, 3, and 5), there was no significant difference between the regression lines; and for one (Subject 4), the intercept of the regression for Set $\mathbf{R}$ was higher than that for Set P (although the slope for both sets was 0 ). Only Subject 10 showed a significant difference between the within-set slopes $[F(1,14)=$ $5.51, \mathrm{p}=.034]$. 
Table 1

Breakdown of Components of $\mathbf{C}$ for the Stimulus Figures and Geometric Mean of Magnitude Estimates of Complexity

\begin{tabular}{|c|c|c|c|c|c|c|}
\hline Stimulus & $\begin{array}{c}\text { Number of } \\
\text { Segments }\end{array}$ & $\mathrm{E}\left(\mathrm{n}_{\lambda}\right)^{*}$ & $\mathrm{E}\left(\mathrm{n}_{\boldsymbol{\omega}}\right)^{* *}$ & $E\left(n_{\alpha}\right)$ & $\mathrm{C}$ & $\begin{array}{c}\text { Geom. Mean } \\
\text { Magnitude Est. }\end{array}$ \\
\hline \multicolumn{7}{|c|}{ Set $\mathbf{R}$ (Rectangular Partitions) } \\
\hline R1 & 18 & 113 & 196 & 86 & 265 & 21.219 \\
\hline R2 & 20 & 108 & 206 & 93 & 270 & 19.160 \\
\hline R3 & 26 & 145 & 243 & 118 & 344 & 14.539 \\
\hline R4 & 26 & 143 & 255 & 110 & 338 & 30.988 \\
\hline R5 & 26 & 160 & 243 & 136 & 377 & 24.321 \\
\hline R6 & 37 & 246 & 328 & 136 & 492 & 23.637 \\
\hline R7 & 44 & 249 & 406 & 164 & 548 & 45.777 \\
\hline R8 & 51 & 291 & 473 & 197 & 646 & 57.217 \\
\hline R9 & 67 & 401 & 598 & 256 & 856 & 62.635 \\
\hline \multicolumn{7}{|c|}{ Set $P$ (Polygonal Partitions) } \\
\hline P1 & 19 & 141 & 215 & 78 & 433 & 41.339 \\
\hline P2 & 19 & 112 & 224 & 114 & 450 & 73.859 \\
\hline P 3 & 29 & 189 & 312 & 130 & 632 & 150.680 \\
\hline P4 & 32 & 216 & 282 & 122 & 620 & 81.538 \\
\hline P5 & 24 & 127 & 289 & 76 & 492 & 44.979 \\
\hline P6 & 45 & 289 & 470 & 180 & 817 & 379.140 \\
\hline P7 & 38 & 215 & 423 & 156 & 794 & 189.040 \\
\hline P8 & 54 & 362 & 542 & 222 & 1127 & 199.340 \\
\hline P9 & 60 & 416 & 583 & 222 & 1222 & 275.950 \\
\hline
\end{tabular}

\section{DISCUSSION}

C underestimates the complexity of the polygonal partitions or overestimates the complexity of the rectangular partitions. Interestingly, Vitz and Todd (1971, p. 218) noted that one of their figures "which consists of entirely vertical and horizontal lines seems to have a predicted value that is slightly too large.... There is considerable evidence that humans perceive vertical and horizontal lines with greater efficiency than lines at other orientations.... The model assumes all orientations are sampled with equal probability and this may need correct." It should be noted that the problem of overestimation of complexity of purely rectangular forms cannot be solved by Vitz and Todd's suggestion. Assigning probabilities differentially to vertical and horizontal lines as compared to lines of other orientations would give differential weight to vertical and horizontal lines in mixed figures, but would not change the number of samples required to exhaust all the elements of purely rectangular or nonrectangular figures. Suppose, however, that the visual system can process a vertical or horizontal line more quickly than a line of some other orientation. Then, if one postulated that the complexity-assessment mechanism gauges the latency of samples, ${ }^{2}$ complexity could be easily related to orientation.

Furthermore, the evidence at hand does not allow us to say whether the effect is due to the verticality and horizontality of the lines in the stimuli. It is quite possible that the crucial variables are the parallelism and perpendicularity of the lines.
The main problems with the model, however, are its lack of parsimony and a paucity of behavioral evidence for the stratified sampling process it

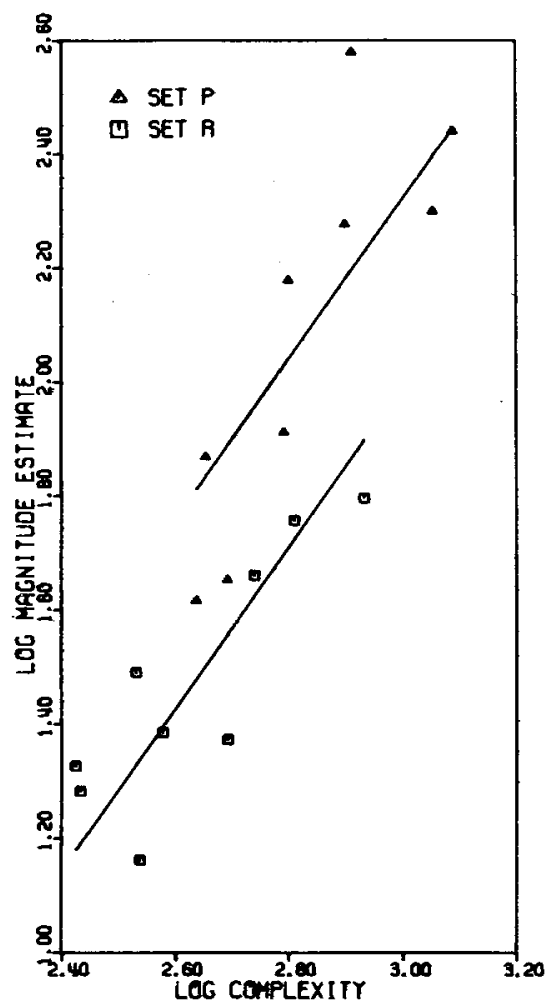

Figure 2. Complexity estimates as a function of Vitz and Todd's (1971) index $C\left(\log _{10}\right)$. The equations of the fitted lines are: Set $R, m=.0058 C^{1.409} ;$ Set $P, m=.0125 C^{1.409}$. 
Table 2

Stepwise Multiple Regression Using $C$ and Adding Partition Type (p) Compared to Simple Regression Using the N/S Predictor

\begin{tabular}{|c|c|c|c|c|c|c|}
\hline \multirow[b]{2}{*}{ Subject } & \multicolumn{4}{|c|}{ Stepwise Multiple Regression } & \multicolumn{2}{|c|}{ Simple Regression } \\
\hline & $\mathbf{R}_{\mathbf{C}}^{2}$ & $\begin{array}{c}F(1,16) \text { for } \\
\text { C Alone }\end{array}$ & $\mathbf{R}_{\mathbf{C p}}^{2}$ & $\begin{array}{c}F(1,15) \text { for } \\
\text { Adding } p\end{array}$ & $\mathrm{R}_{\mathrm{N} / \mathrm{S}}^{2}$ & $\begin{array}{c}F(1,16) \text { for } \\
N / S\end{array}$ \\
\hline 1 & .5748 & $21.63^{* *}$ & .8193 & $20.30 * *$ & .7716 & $54.05^{* *}$ \\
\hline 2 & .5598 & $20.35^{* *}$ & .5857 & .94 & .5658 & $20.85^{* *}$ \\
\hline 3 & .5619 & $20.52 * *$ & .6194 & 2.27 & .6274 & $26.94 * *$ \\
\hline 4 & 0 & 0 & .7695 & $50.08^{* *}$ & .0865 & 1.51 \\
\hline 5 & .3469 & $8.50^{*}$ & .3844 & .91 & .2384 & 5.01 \\
\hline 6 & .6825 & $34.39 * *$ & .9370 & $60.60^{* *}$ & .9242 & $194.96 * *$ \\
\hline 7 & .6186 & $25.95 * *$ & .8445 & $21.79 * *$ & .8366 & $81.90^{* *}$ \\
\hline 8 & .5822 & $22.30 * *$ & .8846 & $39.31 * *$ & .8264 & $76.19 * *$ \\
\hline 9 & .6977 & $36.93^{* *}$ & 9056 & $33.04 * *$ & .8937 & $134.47^{* *}$ \\
\hline 10 & .6288 & $27.10^{* *}$ & .7455 & $6.88^{*}$ & .7453 & $46.83^{* *}$ \\
\hline Geometric Mean & .7493 & $47.82^{* *}$ & .8620 & $12.25 * *$ & .8688 & $105.93^{* *}$ \\
\hline
\end{tabular}

${ }^{*} p<.05$

hypothesizes. Indeed, a different predictor, which suggests a much simpler and no less plausible process model, can account for our data better than $C$.

The predictor we propose (labeled N/S) has just two parameters: the number of line segments in the figure and the measure of angular symmetry described by Vitz and Todd (1971, pp. 213-215). According to Vitz and Todd's method, the measure of symmetry is 3.00 for the rectangular partitions and 1.00 for the

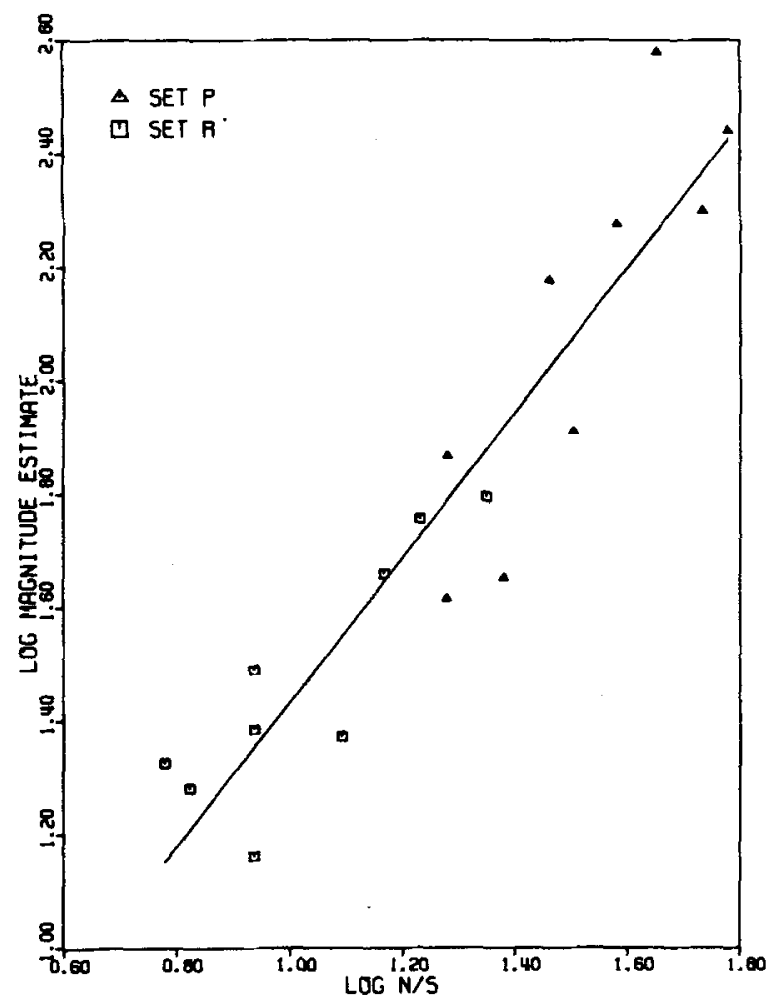

Figure 3. Complexity estimates as a function of the $\mathrm{N} / \mathrm{S}$ index $\left(\log _{10}\right)$. The equation of the fitted line is $\mathrm{m}=1.4431(\mathrm{~N} / \mathrm{S})^{1.275}$. polygonal partitions. Table 2 shows that, after a $\log -\log$ transformation of the $N / S$ predictor and of the geometric mean of the magnitude estimations, a linear regression function accounts for $\mathrm{R}_{\mathrm{N}}^{2} / \mathrm{S}=.8688$ of the variance. Figure 3 graphs on a $\log -\log$ plot the geometric mean of the magnitude estimates of complexity as a function of $N / S$. The $N / S$ predictor does better than $C$ for 9 of the 10 subjects. Furthermore, Wilcoxon's signed rank test applied to the 10 pairs of coefficients of determination $\left(R_{C}^{2}\right.$ and $\mathrm{R}_{\mathrm{N} / \mathrm{S}}^{2}$ appearing in Table 2) shows that the $\mathrm{N} / \mathrm{S}$ predictor is significantly better than $\mathrm{C}(\mathrm{W}=47, \mathrm{~N}=$ $10, p<.01)$.

We also applied the $\mathrm{N} / \mathrm{S}$ predictor to the data obtained by Vitz and Todd in their Experiments $I$ and II on closed polygonal forms. C accounts for $94.09 \%$ and $92.16 \%$ of the variance in the mean rank of the judged complexity, whereas the $\mathrm{N} / \mathrm{S}$ predictor accounts only for $80.91 \%$ and $79.40 \%$ of the same variance. Tests of the differences between these coefficients of determination show that in this case $\mathrm{C}$ is the better predictor $[F(2,23)=25.65$ and $F(2,23)$ $=10.58$, both $\mathrm{p}<.01]$. Thus, it would appear that either (a) $C$ does better for closed polygonal forms than for the type of forms generated for the present experiment, or (b) it does better for simpler forms, such as those tested by Vitz and Todd (values of $C$ ranging from 4.70 to 261.01), than for more complex forms, such as those used here (values of $\mathrm{C}$ ranging from 265 to 1222 ).

Garner (1974) has shown that the goodness (which may be thought of as being the inverse of complexity) of a stimulus is related to the size of the inferred subset produced by the stimulus. We may conjecture that the inferred set of rectangular partitions is perceived as being a proper subset of the inferred set of polygonal partitions. We would expect, therefore, rectangular partitions to appear less complicated. This is, indeed, the case. It is unlikely, however, that 
subjects construct just to inferred sets in performing their task. If they were doing that, we would not find a high correlation between their judgments of complexity and $C$ within each set of stimuli. It follows that subjects can perform a much finer division of the set of all partitions of the square. How they perform this and similar tasks remains a central puzzle of pattern-recognition research, which Vitz and Todd's approach does not seem to have solved.

\section{REFERENCES}

GARNER, W. R. The processing of information and structure. New York: Halstead-Wiley, 1974.

Green, D. M., \& LuCE, R. D. Counting and timing mechanisms in auditory discrimination and reaction time. In D. H. Krantz, R. C. Atkinson, R. D. Luce, \& P. Suppes (Eds.), Contemporary developments in mathematical psychology (Vol. 2). San Francisco: Freeman, 1974.

Terwilliger, R. F. Pattern complexity and affective arousal. Perceptual and Motor Skills, 1963, 17, 387-395.

ViTz, P. C. Affect as a function of a stimulus variation. Journal of Experimental Psychology, 1966, 71, 74-79.

Vitz, P. C., \& ToDd, T. C. A model of the perception of simple geometric figures. Psychological Review, 1971, 78. 207-228.

\section{NOTES}

1. We are grateful to Paul C. Vitz for supplying the program.

2. This would change the model from a counting model to a timing or clocking model (for a discussion of the difference, see Green \& Luce. 1974, pp. 386-393).

(Received for publication January 10, 1975: revision received May 20, 1975.)

\section{ERRATUM}

Lappin, Joseph S., \& Preble, Laurence D. A Demonstration of Shape Constancy. Perception \& Psychophysics, $1975,5,439-444$. The table on page 441 was incorrect. The correct table appears below.

Table 1

The Correspondences of Judged Angles With Actual Projective and Objective Angles

\begin{tabular}{|c|c|c|c|c|c|c|c|c|c|c|c|}
\hline & \multirow{2}{*}{$\begin{array}{l}\text { Judgment } \\
\text { Condition }\end{array}$} & \multirow{2}{*}{$\begin{array}{l}\text { Actual } \\
\text { Angle }\end{array}$} & \multicolumn{8}{|c|}{ Subjects } & \multirow[b]{2}{*}{ Average } \\
\hline & & & 1 & 2 & 3 & 4 & 5 & 6 & 7 & 8 & \\
\hline \multirow{9}{*}{$\begin{array}{l}\text { Absolute } \\
\text { Deviation } \\
\text { (Degrees) } \dagger\end{array}$} & \multirow{2}{*}{$\begin{array}{l}\text { Upright, } \\
\text { Objective }\end{array}$} & Projective & 17.8 & 22.5 & $\begin{array}{c}16.1 \\
* *\end{array}$ & 16.9 & 15.0 & $\begin{array}{r}19.2 \\
* * *\end{array}$ & 16.7 & 17.2 & 17.7 \\
\hline & & Objective & 9.4 & 8.6 & 5.6 & 10.3 & 12.8 & 6.4 & 11.7 & 6.1 & 8.9 \\
\hline & \multirow{2}{*}{$\begin{array}{l}\text { Upright, } \\
\text { Projective }\end{array}$} & Projective & 19.4 & 12.5 & 14.4 & 16.1 & 15.0 & $\begin{array}{c}18.3 \\
* *\end{array}$ & $\begin{array}{c}15.6 \\
* *\end{array}$ & 12.8 & 15.5 \\
\hline & & Objective & 12.2 & 13.6 & 8.9 & 5.6 & 8.3 & 5.0 & 7.2 & 10.0 & 8.8 \\
\hline & \multirow{3}{*}{$\begin{array}{l}\text { Inverted, } \\
\text { Objective }\end{array}$} & Projective & 17.8 & 19.4 & 13.6 & 12.5 & 15.8 & 16.7 & 15.8 & 17.2 & 16.1 \\
\hline & & & $\begin{array}{c}* * \\
556\end{array}$ & $* *$ & $10 ?$ & 86 & & $* *$ & ** & $* *$ & 76 \\
\hline & & oojective & 35.0 & & 10.3 & 8.0 & 9.1 & & & & 1.0 \\
\hline & \multirow{2}{*}{$\begin{array}{l}\text { Inverted, } \\
\text { Projective }\end{array}$} & Projective & 16.7 & 14.7 & 10.8 & 13.9 & 13.6 & 16.7 & 13.1 & 15.6 & 14.4 \\
\hline & & Objective & 7.8 & 10.3 & 11.9 & 8.3 & 12.5 & 7.8 & 9.2 & 11.1 & 9.9 \\
\hline \multirow{8}{*}{$\mathbf{r}^{2}$} & Upright, & Projective & .00 & .03 & .03 & .01 & .08 & .01 & .00 & .00 & .02 \\
\hline & Objective & Objective & .40 & .62 & .72 & .28 & .23 & .77 & .36 & .72 & .52 \\
\hline & Upright, & Projective & .00 & .27 & .08 & .02 & .12 & .02 & .05 & .13 & .09 \\
\hline & Projective & Objective & .31 & .28 & .37 & .77 & .62 & .82 & .65 & .43 & .53 \\
\hline & Inverted, & Projective & .00 & .00 & .15 & .13 & .02 & .00 & .02 & .00 & .04 \\
\hline & Objective & Objective & .79 & .77 & .34 & .54 & .33 & .63 & .64 & .65 & .59 \\
\hline & Inverted, & Projective & .03 & .09 & .31 & .17 & .18 & .03 & .21 & .06 & .14 \\
\hline & Projective & Objective & .58 & .48 & .22 & .58 & .35 & .62 & .41 & .35 & .45 \\
\hline
\end{tabular}

